\title{
ON PURITY OF INERTIA
}

\author{
DAVID HARBATER
}

(Communicated by Louis J. Ratliff, Jr.)

\begin{abstract}
This paper considers the phenomenon of inertia groups jumping at regular points of the branch locus of a cover, especially in mixed characteristic. After giving conditions under which this cannot occur, these ideas are applied to the problem of obtaining groups as Galois groups of unramified covers of the affine line in finite characteristic.
\end{abstract}

\section{INTRODUCTION}

In the process of proving resolution of singularities for surfaces in characteristic $p$ [Ab1], Abhyankar first noticed that the classical proof [Ju] over the complex numbers cannot be carried over. The difficulty was studied in [Ab2], where it was referred to as the phenomenon of "local splitting of a simple branch variety by itself." That is, in characteristic $p$, the ramification locus of a normal cover of a regular variety can be singular even at a point lying over a regular point of the branch locus [Ab2, $\S 3$, Examples 1, 2, 5]. By taking linear sections of these examples, Abhyankar obtained examples [Ab3] of unramified covers of the affine line in characteristic $p$ with nonsolvable Galois group.

Motivated by these examples, Abhyankar conjectured in [Ab3] that a finite group $G$ occurs as a Galois group of an unramified cover of a given characteristic $p$ curve of genus $g$ with $r$ points deleted if and only if the maximal prime-to- $p$ quotient $G^{\prime}$ of $G$ occurs, and that this is the case if and only if $G^{\prime}$ occurs over the corresponding curve in characteristic 0 . (Thus $G$ is conjectured to occur over the affine line if and only if it is a "quasi- $p$-group," i.e., generated by its Sylow $p$-subgroups.) The latter part of this conjecture was later proven by Grothendieck [Gr2]. The former part remains unknown, but recently Serre [Se] has shown it to be true in the case that $G$ is solvable. Also, Abhyankar has recently computed (cf. [Ab4]) the Galois groups of various covers of the affine line (including several from [Ab3]), and found that most alternating and symmetric groups occur.

Received by the editors October 27, 1989 and, in revised form, August 1, 1990.

1980 Mathematics Subject Classification (1985 Revision). Primary 14E22, 14G20; Secondary $14 \mathrm{E} 20,14 \mathrm{H} 25$.

Key words and phrases. Purity, inertia, branched cover, fundamental group. 
The present paper studies the "local splitting" phenomenon especially in the mixed characteristic case, and uses it to obtain information about the fundamental group of the affine line in characteristic $p$ (somewhat analogously to the use of [Ab2] in [Ab3]). In particular, we consider the question of when the ramification locus of a cover can be locally reducible over a regular point of the branch locus. While purity of branch locus holds for covers even over arbitrary regular rings (cf. [Na, 41.1]), in this situation there is a failure of "purity of inertia," in the sense that the inertia groups jump at a point of codimension $\geq 2$ in the base space, at a regular point of the branch locus. An example of this phenomenon in characteristic $p$ appeared as [Ab2, §3, Example 5]. Here we give examples in mixed characteristic, as well as showing that under certain hypotheses the phenomenon cannot occur. In addition, we discuss a class of families of $p$-adic covers having the property that each member of the family either provides an example in which "purity of inertia" fails, or else yields (after taking a section) an unramified cover of the affine line in characteristic $p$ having rather general Galois group of the type conjectured by Abhyankar to occur.

To fix terminology, by a cover we will mean a morphism $\pi: Y \rightarrow X$ that is finite and generically étale, such that $Y$ is normal and connected and such that $X$ is regular and connected. We will call such a cover Galois with group $G$ provided that the function field of $Y$ is Galois with group $G$ over the function field of $X$. Thus $G$ acts on $Y$ over $X$. As usual, we say that $g \in G$ is in the decomposition group at a point $y$ of $Y$ if $g(y)=y$, and say that such a $g$ is in the inertia group at $y$ if moreover $g \equiv$ identity $\left(\bmod m_{y}\right)$ in $O_{y}$. Thus $\pi$ is ramified at a point $y$ if and only if the inertia group at $y$ is nontrivial. The (reduced) set of such $y$ is the ramification locus, and its (reduced) image in $X$ is the branch locus. Ramification at a point $y$ is tame if the order of the inertia group is not divisible by the characteristic of the residue field at $y$.

\section{SOME PURITY RESULTS}

We begin with some results which show that purity of inertia does hold for a Galois cover provided that appropriate additional hypotheses are assumed.

Proposition 1. Let $\pi: Y \rightarrow X$ be a Galois cover, let $y$ be a ramified point of $Y$, and let $x=\pi(y)$. Assume that $x$ is a regular point of the branch locus $B$. If the ramification is tame at the generic point of the branch component containing $x$, then there is a Zariski neighborhood $N$ of $y$ in the ramification locus such that every point of $N$ has the same inertia group.

Proof. Since $x$ is a regular point of $B, x$ lies on a unique irreducible component $B_{0}$ of $B$. Here $B_{0}$ has codimension 1 in $X$, by purity of branch locus [Na, 41.1]. Since $X$ is regular (say of dimension $d$ ), it follows that there is an affine Zariski neighborhood $U=\operatorname{Spec} R$ of $x$ in which the reduced scheme $B$ is defined by an $f \in R$. Since $B$ is regular at $x$, after shrinking $U$ we may 
assume that $B \cap U=\operatorname{Spec} U /(f)$ is regular (of dimension $d-1$ ) and that $B_{0}$ is the only component of $B$ meeting $U$.

Since the ramification at the generic point of the hypersurface $B_{0}$ is tame, the inertia group is cyclic, say of order $e$. Let $R^{\prime}=R[t] /\left(t^{e}-f\right)$ and $U^{\prime}=\operatorname{Spec} R^{\prime}$. We claim that $U^{\prime}$ is regular at the point $z$ lying over $x \in U$. Namely, if $x$ is of codimension $r$ on $B$, then $O_{B, x}$ has a regular system of parameters $\left(f_{1}, \ldots, f_{r}\right)$. So $O_{U, x}$ has a regular system of parameters $\left(f, f_{1}, \ldots, f_{r}\right)$, and $O_{U^{\prime}, z}$ has a regular system of parameters $\left(t, f_{1}, \ldots, f_{r}\right)$. So $U^{\prime}$ is indeed regular at $z$; and after shrinking $U$ and $U^{\prime}$, we may assume $U^{\prime}$ is regular.

Let $V=\operatorname{Spec} S=\pi^{-1}(U)$ and let $S^{\prime}$ be the normalization of $S \otimes_{R} R^{\prime}$. Then $V^{\prime}=\operatorname{Spec} S^{\prime}$ is unramified over $U^{\prime}$ at the generic point of the pullback of $B$, by Abhyankar's Lemma [ $\mathrm{Gr} 2, \mathrm{X}$, Lemme 3.6]. And $V^{\prime}$ is unramified at every other height 1 prime, since $V$ is unramified at every height 1 prime except $(f)$. By purity of branch locus, $V^{\prime} \rightarrow U^{\prime}$ is unramified. So the inertia groups over $B \cap U$ have order at most $e$. But since inertia can only increase upon specialization, these inertia groups have order exactly $e$, and inertia is constant on components of $\pi^{-1}(B \cap U)$. So $N$ exists.

Proposition 2. Let $\pi: Y \rightarrow X$ be a Galois cover and let $y$ be a ramified point of $Y$. Assume that $y$ lies on exactly one irreducible component of the ramification locus (e.g. if $y$ is a regular point of the ramification locus). Then there is a Zariski neighborhood $N$ of $y$ in the ramification locus such that every point of $N$ has the same inertia group.

Proof. Let $G$ be the Galois group of the cover, let $C$ be the irreducible component of the ramification locus which contains $y$, and let $H \subset G$ be the inertia group at the generic point $c$ of $C$. Let $Z$ be the quotient of $Y$ by $H$, and let $z$ be the image of $y$ in $Z$. Then $Z$ is also normal, and so the induced morphism $\sigma: Z \rightarrow X$ is also a cover. By definition of $H, \sigma$ is unramified at $c$. Also, $\sigma$ is unramified at any point $z^{\prime}$ that is the image of an unramified point $y^{\prime}$ of $\pi$. So the local ring $O_{Z, z}$ dominates $O_{X, x}$ and is unramified in codimension 1. Applying purity of branch locus $[\mathrm{Na}, 41.1]$ to this extension of local rings, we conclude that $O_{Z, z}$ is unramified over $O_{X, x}$. Hence there is a Zariski open neighborhood $M$ of $z$ in $Z$ in which $\sigma$ is unramified. Let $N$ be the inverse image of $M$ in $Y$. After shrinking $N$ we may assume that $C$ is the only ramification component meeting $N$. Thus the inertia group of every ramified point in $N$ is contained in $H$. Since the locus at which $H$ is contained in inertia is closed, it follows that the inertia is exactly $H$ at every ramified point in $N$.

Corollary. Let $O$ be the ring of integers of a p-adic field, and let $X$ be a regular scheme which is projective of relative dimension 1 over $O$. Let $\pi: Y \rightarrow X$ be a Galois cover. If $Y$ is smooth over $O$, then the cover satisfies purity of inertia.

Proof. Let $x \in X$ be a regular point of the branch locus, and let $y \in Y$ lie over $x$. Then $x$ lies on a unique component $C$ of the branch locus. If $C$ is 
horizontal, then the residue characteristic is 0 , and so by Proposition 1, purity of inertia holds there. Otherwise, the component lies in the vertical closed fibre of $X$. By smoothness, the closed fibre of $Y$ is regular, and so $y$ cannot lie on more than one component of the fibre. So $y$ lies on a unique component of the ramification locus, and the conclusion follows by Proposition 2.

\section{RELATIONSHIP TO $\pi_{1}$ AND REDUCTION $\bmod p$}

This section discusses the relationship between the failure of purity of inertia and the failure of reduction $\bmod p$ to be a cover. We consider certain covers of $\mathbf{P}^{1}$ over $p$-adic rings whose horizontal branch components meet the closed fibre only at $x=\infty$, and whose Galois groups $G$ lie in a class of groups that are conjectured [Ab4, $\S 4$, Conjecture 1] to be quotients of $\pi_{1}\left(\mathbf{A}^{1}\right)$ in characteristic $p$. Each of these covers gives rise either to a $G$-Galois cover of $\mathbf{A}^{1}$ in characteristic $p$, or else to a counterexample to purity of inertia. This suggests that by controlling a "jumping," one might be able to construct étale covers of $\mathbf{A}^{1}$ with fairly general Galois group.

Let $K$ be a $p$-adic field, that is, a finite extension of the field $\mathbf{Q}_{p}$, and let $O$ be the ring of integers in $K$, with uniformizer $\omega$ and residue field $k$. Suppose that $K$ contains a primitive $(2 p-2)$ th root of unity $\zeta$, a primitive $p$ th root of unity $\mu$, and an element $\pi=p^{1 /(p-1)}$. Let $x$ be a parameter for $\mathbf{P}^{1}$. We will say that a polynomial $F(x)=1+\sum_{i=0}^{p m} \pi^{p} c_{i} x^{i} \in O[x]$ is standard if all $c_{i} \in O$, and if $c_{p m-1}$ is a unit in $O$ while $c_{p m}$ is not. (Here we permit $c_{p m}=0$.)

Lemma. If $F$ is a standard polynomial over $O$, then there is a Galois cover $Z \rightarrow \mathbf{P}_{O}^{1}$ with group $\mathbf{Z} / p$, where $Z$ is regular, and such that

(i) the generic fibre $Z_{K} \rightarrow \mathbf{P}_{K}^{1}$ is geometrically irreducible and is given generically by $y^{p}=F(x)$; and

(ii) the closed fibre $Z_{k} \rightarrow \mathbf{P}_{k}^{1}$ is geometrically regular and irreducible, and is ramified only over the point at infinity on $\mathbf{P}^{1}$ (where it is totally ramified).

Proof. Write $F(x)=1+(\pi / \zeta)^{p} f(x)$, where $f(x)=\sum_{i=0}^{p m} a_{i} x^{i} \in O[x]$. Expanding the polynomial $\phi(z)=(z+\zeta / \pi)^{p}-(\zeta / \pi)^{p} \in K[z]$, we have that $\phi(z) \in O[z]$, and that $\phi(z) \equiv z^{p}-z(\bmod \pi)$. The extension of $K[x]$ given by $\phi(z)=f(x)$ is isomorphic to that given by $w^{p}=f(x)+(\zeta / \pi)^{p}$ (where $w=z+\zeta / \pi)$, or equivalently to that given by $y^{p}=F(x)$. So it is $\mathbf{Z} / p$-Galois over $K[x]$, since $\mu \in K$.

Let $A=O[x, z] /(\phi(z)-f(x))$. Setting $\bar{x}=x^{-1}, \bar{z}=\bar{x}^{m} z, \bar{f}(\bar{x})=$ $\bar{x}^{p m} f(x)$, and $\bar{\phi}(\bar{z})=\bar{x}^{p m} \phi(z)$, let $\bar{A}=O[\bar{x}, \bar{z}] /(\bar{\phi}(\bar{z})-\bar{f}(\bar{x}))$. Patching $\operatorname{Spec} A$ to $\operatorname{Spec} \bar{A}$, we obtain $Z \rightarrow \mathbf{P}_{O}^{1}$. The branch locus of $Z \rightarrow \mathbf{P}_{O}^{1}$ is given by $(F(x))$, and thus meets the closed fibre $\mathbf{P}_{k}^{1}$ precisely at $x=\infty$. So $Z$ is regular over $\mathbf{A}_{k}^{1}$. Also, $\bar{f}(\bar{x}) \equiv a_{p m-1} \bar{x}(\bmod (\pi, \bar{x}))$ and $\bar{\phi}(\bar{z}) \equiv \bar{z}^{p}-\bar{x}^{(p-1) m} \bar{z}$ $(\bmod \pi)$. So $\{\omega, \bar{z}\}$ is a regular system of parameters for $Z$ at the point $(\omega, \bar{x}, \bar{z})$ lying over the point $(x=\infty)$ on $\mathbf{P}_{k}^{1}$, and so $Z$ is regular there, too. 
Thus $Z$ is regular at every closed point, and hence is regular. Similarly its fibre $Z_{k}$ is regular (indeed, geometrically regular). Moreover $Z_{K}$ is geometrically irreducible. Hence by the properness of $Z$ over $O$ and the integrality of $Z$, $Z_{k}$ is geometrically connected, by [Gr1, Corollary 4.3.12]. Since $Z_{k}$ is also geometrically regular, it is geometrically irreducible.

If $Z$ is as in the lemma, we will call $Z \rightarrow \mathbf{P}_{O}^{1}$ a standard p-cover.

Remarks. (a) The branch loci of standard $p$-covers can be arbitrarily large, by taking $\operatorname{deg} F \gg 0$. Thus every branched cover can be deformed to a cover whose branch locus is contained in that of a standard $p$-cover.

(b) By varying the coefficients of a standard polynomial, we may obtain a family of standard $p$-covers with nonconstant branch locus. Over the closed fibre we obtain a geometrically nonconstant family of branched covers of $\mathbf{P}_{k}^{1}$ having constant branch locus $x=\infty$. Here, the lifting to the family over $O$ can be interpreted as providing a geometric "explanation" for the fact that covers in characteristic $p$ may be deformed without moving the branch points-viz. that branch points are indeed moving, though over $O$, not $k$.

If a group $G$ is generated by elements $g_{1}, \ldots, g_{n}$ of order $p$, then there is a cover of $\mathbf{P}^{1}$ whose inertia groups all have order $p$, and which is branched at a prescribed set of $2 n$ points. Namely, over the algebraic closure of $\mathbf{Q}_{p}$, $\pi_{1}\left(\mathbf{P}^{1}-\left\{Q_{1}, \ldots, Q_{2 n}\right\}\right)$ is the profinite group on generators $\gamma_{1}, \ldots, \gamma_{2 n}$ subject to the relation $\gamma_{1} \cdots \gamma_{2 n}=1$ [Gr2, XIII, Corollaire 2.12]; now take $\pi_{1} \rightarrow G$ by $\gamma_{2 i-1} \mapsto g_{i}$ and $\gamma_{2 i} \mapsto g_{i}^{-1}$ in order to obtain the cover. Being of finite type, this cover is defined over some $p$-adic field $K$.

Proposition 3. Suppose that $G$ is a finite group which has no nontrivial normal p-subgroups, and which is generated by elements of order $p$. Let $Y_{K} \rightarrow \mathbf{P}_{K}^{1}$ be a Galois cover with group $G$ whose inertia subgroups are all of order $p$, and whose branch locus is strictly contained in that of $Z_{K} \rightarrow \mathbf{P}_{K}^{1}$, for some standard p-cover $Z \rightarrow \mathbf{P}_{O}^{1}$. Let $Y \rightarrow \mathbf{P}_{O}^{1}$ be the normalization of $\mathbf{P}_{O}^{1}$ in $Y_{K}$. Then, possibly after enlarging $K$, either

(a) the normalization of the closed fibre of $Y \rightarrow \mathbf{P}_{O}^{1}$ is a geometrically irreducible G-Galois cover of $\mathbf{P}^{1}$ that is ramified only over the point at infinity; or

(b) the pullback of $Y \rightarrow \mathbf{P}_{O}^{1}$ by the regular cover $Z \rightarrow \mathbf{P}_{O}^{1}$ has normalization $Y^{\prime} \rightarrow Z$ which is ramified precisely over the closed fibre, and violates purity of inertia.

Proof. Let $Y_{k}$ be the fibre over $\mathbf{P}_{k}^{1}$ and let $e$ be the ramification index of $Y$ over the generic point of the closed fibre. Write $e=p^{v} s$, where $s$ is prime to $p$. After replacing $K$ by $K\left(\omega^{1 / s}\right)$ and similarly enlarging $O$, by Abhyankar's Lemma [Gr2, X, Lemme 3.6] we may assume that $e$ is a power of $p$.

To prove the result, it suffices to show that if $e=1$ then (a) holds and if $e>1$ then (b) holds. 
Let $Y_{K}^{\prime}$ be the normalization of the fibre product of $Z_{K}$ and $Y_{K}$ over $\mathbf{P}_{K}^{1}$. Since $Z_{K}$ and $Y_{K}$ are Galois and have no common subcovers (inasmuch as $Z_{K} \rightarrow \mathbf{P}^{1}$ is a $\mathbf{Z} / p$-cover which is branched at more points than $Y_{K} \rightarrow \mathbf{P}^{1}$ is), it follows that $Y_{K}^{\prime}$ is geometrically irreducible. Let $Y^{\prime}$ be the normalization of $Y_{K}^{\prime}$ over $\mathbf{P}_{O}^{1}$, and let $Y_{k}^{\prime}$ be the fibre over $k$. Now $Y^{\prime}$ is proper and dominant over $O ; K$ is algebraically closed in the function field of $Y^{\prime}$ (since $Y_{K}^{\prime}$ is geometrically irreducible); $O$ is a regular domain; and $Y^{\prime}$ is a noetherian integral scheme. So by [Gr1, Corollaire 4.3.12], it follows that the closed fibre $Y_{k}^{\prime}$ is geometrically connected. Also, $Y_{K}^{\prime} \rightarrow Z_{K}$ is unramified by Abhyankar's Lemma, since the branch locus of $Y_{K}$ is contained in that of $Z_{K}$, and since both have $p$-cyclic ramification.

Now suppose that $e=1$, that is, that $Y_{k} \rightarrow \mathbf{P}_{k}^{1}$ is generically unramified. Then $Y^{\prime} \rightarrow Z$ is unramified in codimension 1 . Since $Z$ is regular, purity of branch locus [Na, 41.1] applies; so $Y^{\prime} \rightarrow Z$ is étale. So $Y_{k}^{\prime}$ is an étale cover of the regular scheme $Z_{k}$, and hence is regular and thus locally irreducible. Since $Y_{k}^{\prime}$ is also geometrically connected, it is geometrically irreducible. Hence the same is true for $Y_{k}$, and (a) follows.

Now instead suppose that $e>1$, so that the branch locus of $Y^{\prime} \rightarrow Z$ is the closed fibre of $Z$, which is regular. We claim that (b) holds. For if not, then the inertia groups are constant over the branch locus, and hence $Y_{k}^{\prime}$ is locally irreducible. Since $Y_{k}^{\prime}$ is geometrically connected, it is geometrically irreducible. So the localization of $Y^{\prime}$ at the generic point of $Y_{k}^{\prime}$ is a discrete valuation ring which is finite and generically separable over the localization of $Z$ at the generic point of $Z_{k}$; moreover this extension is Galois with group $G$. So the inertia group of this extension is normal in $G$. But the order of this inertia group is $e$, which is a power of $p$. Since $e>1$ and $G$ has no nontrivial normal $p$-subgroups, this is a contradiction.

Note that the proposition applies in particular to simple groups of order divisible by $p$.

Remark. The hypothesis of strict containment is not crucial. Specifically, suppose that the branch locus of $Y_{K} \rightarrow \mathbf{P}_{K}^{1}$ is instead equal to that of $Z_{K} \rightarrow \mathbf{P}_{K}^{1}$. If $Y_{K} \rightarrow \mathbf{P}_{K}^{1}$ does not dominate $Z_{K} \rightarrow \mathbf{P}_{K}^{1}$, then arguing as before we conclude that again either (a) or (b) must hold. On the other hand if $Y_{K} \rightarrow \mathbf{P}_{K}^{1}$ dominates $Z_{K} \rightarrow \mathbf{P}_{K}^{1}$, then either (a) holds, or else we have

$$
\begin{aligned}
& \text { (b) } \quad Y \rightarrow Z \text { is ramified precisely over the } \\
& \text { closed fibre, and violates purity of inertia. }
\end{aligned}
$$

Note that (b) ${ }^{\prime}$ cannot occur if $Y \rightarrow Z$ has degree prime to $p$.

In general, under the hypotheses of the above proposition, it would be interesting to know when (a) and (b) respectively occur. (Note that (a) must occur if the hypothesis of the corollary to Proposition 2 is satisfied.) For a group $G$ 
as in the proposition, if it were known that there exists a choice of $Y_{K}$ yielding (a), then $G$ would be a quotient of $\pi_{1}\left(\mathbf{A}^{1}\right)$ in characteristic $p$.

\section{EXAMPLES}

We conclude with some examples of Proposition 3, each of which leads either to a Galois cover of $\mathbf{P}^{1}$ branched only at infinity, or else yields a counterexample to purity of inertia.

Example $1\left(G=S_{3}, p=2\right)$. Let $O$ be a finite extension of $\mathbf{Z}_{2}$, and let $Y \rightarrow \mathbf{P}_{O}^{1}$ be the Galois closure of the cover of $\mathbf{P}_{O}^{1}$ given generically by $y^{3}-$ $x y+c=0$, where $c \in O$. Thus $Y$ is a Galois cover with group $S_{3}$, and the ramification index over any horizontal branch component is 2 . Over the $x$-patch the ramification locus is given by $x=3 y^{2}$, or equivalently by $-2 y^{3}+c=0$, and the branch locus is given there by $4 x^{3}=27 c^{2}$. In addition, $Y \rightarrow \mathbf{P}_{O}^{1}$ is branched over $x=\infty$. Thus the horizontal branch components meet the vertical fibre only at infinity, if and only if 2 does not divide $c$ in $O$. Assuming $2 \nmid c$, there are two cases:

(i) If $c$ is a unit in $O$, then $Y$ is generically unramified over the closed fibre. The horizontal part of the branch locus is equal to that of a standard $p$-cover, given by $F(x)=1-4 x^{3} / 27 c^{2}$. And indeed, this standard cover is the unique $\mathrm{Z} / 2$-subcover of $Y$. As in the remark following Proposition 3 (and cf. the corollary to Proposition 2), condition (a) holds; that is, $Y_{k} \rightarrow \mathbf{P}_{k}^{1}$ is a Galois cover with group $S_{3}$ and branch locus $(x=\infty)$.

(ii) If $c$ is not a unit, then the entire fibre $\mathbf{P}_{k}^{1}$ is contained in the branch locus, and the fibre $Y_{k}$ is reducible. Indeed, $Y_{k}$ has three irreducible components, and the inertia group at the generic point of each has order 2. These three components meet over the (regular) point $(x=0)$ on the fibre $\mathbf{P}_{k}^{1}$, and so the inertia there is $S_{3}$. So purity of inertia fails.

In particular, consider the case in which $c=\omega^{2}$, where $\omega=2^{1 / 3} \in O$. One may check directly that $O[x, y] /\left(y^{3}-x y+\omega^{2}\right)$ is a normal domain; that the branch locus has two components, viz. $B_{1}=(\omega)$ and $B_{2}=\left(\omega^{2} x^{3}-27\right)$, which do not meet on the finite patch, and which are each regular; and that on the finite patch, the inertia group of $Y \rightarrow \mathbf{P}^{1}$ has order 2 at every point of $B_{1}$ other than at $(\omega, x)$, where inertia is all of $S_{3}$. Moreover, the horizontal branch locus is strictly contained in the branch locus of the standard $p$-cover $Z$ given generically by taking a square root of

$$
F(x)=\left(2^{2 / 3} x^{3}-27\right)\left(2^{4 / 3} x^{6}-2^{2 / 3} x^{3}+1\right) .
$$

The normalization $Y^{\prime}$, of the pullback of $Y$ by $Z$, also violates purity of inertia over the point $(x=0)$ on the closed fibre. This illustrates (b) in Proposition 3.

Example $2\left(G=S_{n}, n\right.$ odd,$\left.p=2\right)$. Consider the cover of $\mathbf{P}^{1}$ over $\mathbf{Q}_{2}$ given by $y^{n}-a x^{s} y^{n-s}+1=0$, where $s$ is odd, $1 \leq s<n$, and $a \in \mathbf{Z}_{2}$. One checks 
that for all but finitely many choices of a unit $a \in \mathbf{Z}_{2}$, this is a simple cover of $\mathbf{P}^{1}$ of degree $n$ (i.e., each ramified fibre has exactly $n-1$ points), and its branch locus $\left(4(n-2)^{(n-2)} a^{n} x^{s n}-n^{n}\right)$ is equal to that of a standard cover. Moreover, the Galois closure of this cover has group $S_{n}$, since any transitive subgroup of $S_{n}$ generated by transpositions is all of $S_{n}$ (by [Me, $\S 4$, Lemme 2 ; $\mathrm{Hu}$, Satz 4.5]). Since $a$ is a unit, the reduction modulo 2 of the integral closure over $\mathbf{Z}_{2}$ is generically separable. So by the remark after Proposition 3, the reduction mod 2 defines an $S_{n}$-Galois cover of the line in characteristic 2, ramified only at infinity. Note that this agrees with [Ab4, §10, Example I.5], which was computed via arduous characteristic 2 calculations.

Example $3\left(G=A_{5}, p=3\right)$. Consider the cover of $\mathbf{P}^{1}$ over a finite extension of $\mathbf{Q}_{3}$, given by

$$
y^{5}-5^{7 / 5} x^{2} y^{3}+2^{-2} \cdot 3^{2} \cdot 5^{9 / 5} x^{4} y+3^{15 / 8} \cdot 5 x^{10}+3^{9 / 8} \cdot 5=0 .
$$

This cover has degree 5; has branch locus $\left(3^{3 / 2} x^{20}+1\right)$, which is equal to that of a standard cover; and has the property that each ramified fibre contains a single ramified point, of ramification index 3. Thus the Galois closure of this cover has group $A_{5}$, since any transitive subgroup of $S_{n}$ generated by 3-cycles is equal to $A_{n}$ [Me, $\S 4$, Lemme 1]. Since $A_{5}$ has no nontrivial quotients, the remark after Proposition 3 implies that either condition (a) or condition (b) must hold. One verifies directly that in this example, it is condition (b) which holds. It would be interesting to see if this example could be deformed to a family (analogous to those in Examples 1 and 2) in which the generic member satisfies condition (a) and thus would give rise to $A_{5}$-Galois unramified covers of the affine line in characteristic 3.

\section{ACKNOWLEDGMENT}

I wish to thank Sybilla Beckmann, Ching-li Chai, Steven Diaz, Ron Donagi, and Leila Schneps for helpful comments.

\section{REFERENCES}

[Ab1] S. Abhyankar, Local uniformization on algebraic surfaces over ground fields of characteristic $p \neq 0$, Ann. of Math. 63 (1956), 491-526.

[Ab2] - On the ramification of algebraic functions, Amer. J. Math. 77 (1955), 575-592.

[Ab3] _ Coverings of algebraic curves, Amer. J. Math. 79 (1957), 825-856.

[Ab4] _ Galois theory of the line in nonzero characteristic, manuscript.

[Gr1] A. Grothendieck, Eléments de géométrie algébrique III (EGA III), $1^{\mathrm{e}}$ partie, Inst. Hautes Études Sci. Publ. Math. 11 (1961).

[Gr2] _-, Revêtements étales et groupe fondamental (SGA 1), Lecture Notes in Math., vol. 224, Springer, New York, 1971.

[Hu] B. Huppert, Endliche Gruppen I, Springer-Verlag, Heidelberg, 1967.

[Ju] H. W. E. Jung, Darstellung der Funktionen eines algebraischen Körpers zweier unabhängigen Veränderlichen in der Umgebung einer Stelle, J. für Math. 133 (1908), 289-314. 
[Me] J.-F. Mestre, Extensions régulières de $\mathbf{Q}(T)$ de groupe de Galois $\tilde{A}_{n}$, J. Algebra 131 (1990), 483-495.

[Na] M. Nagata, Local rings, Interscience, New York, 1962.

[Se] J.-P. Serre, Construction de revêtements étales de la droite affine en caractéristique $p$, Comptes Rendus 311 (1990), 341-346.

Department of Mathematics, University of Pennsylvania, Philadelphia, PennsylvaNIA $19104-6395$ 